\title{
Experiencias y significados sobre el fenómeno de las drogas en estudiantes de enfermería
}

\author{
María de los Ángeles Meza-Benavides ${ }^{1}$ \\ Antonia Regina Ferreira Furegato ${ }^{2}$
}

\begin{abstract}
Este estudio procuró identificar los significados y experiencias de estudiantes de enfermería sobre el fenómeno de las drogas. La recolección de la información se hizo mediante entrevista semi-estructurada. El análisis se realizó mediante 5 categorías temáticas. De los 40 sujetos de la muestra, con edad entre 19 y 24 años, 28 fueron mujeres, 3 nunca recibieron informaciones sobre drogas, y la mayoría había tenido experiencias con consumidores o habían consumido. Este problema, en Costa Rica, está asociado a la estigmatización y al tráfico. Reconocen que la responsabilidad es de todos, mientras identifiquen contradicción del Estado consintiendo la publicidad de las empresas tabacaleras y su complicidad con fábricas de licores. Reconocen la importancia de la prevención y consideran que la forma de abordar el problema no es adecuada. Les gustaría que el tema se incorporara en sus estudios enseñando estrategias de cómo actuar y relacionarse con las personas que consumen drogas.
\end{abstract}

Descriptores: Drogas Diseñadas; Enfermería; Estudiantes de Enfermería; Enseñanza.

\footnotetext{
${ }^{1}$ Licenciada en Enfermería en Salud Mental y Psiquiatría, Magíster Scientae en Ciencias de la Enfermería. Profesora, Escuela de Enfermería, Universidad de Costa Rica, San José, Costa Rica. E-mail: marymeza1@yahoo.com.

2 Enfermera, Doctora en Enfermería. Profesor Titular, Escola de Enfermagem de Ribeirão Preto, Universidade de São Paulo, Centro Colaborador de la OMS para el Desarrollo de la Investigación en Enfermería, SP, Brasil. E-mail: furegato@eerp.usp.br.
}

Correspondencia:

Antonia Regina Ferreira Furegato

Universidade de São Paulo. Escola de Enfermagem de Ribeirão Preto

Departamento de Enfermagem Psiquiátrica e Ciências Humanas

Av. dos Bandeirantes, 3900

Bairro: Monte Alegre

CEP: 14040-902 Ribeirão Preto, SP, Brasil

E-mail: furegato@eerp.usp.br 


\section{Experiências e significados sobre o fenômeno das drogas entre estudantes de enfermagem}

Este estudo procurou identificar significados e experiências de estudantes de enfermagem sobre o fenômeno das drogas. Utilizou-se entrevista semiestruturada para a coleta dos dados. A análise considerou cinco categorias temáticas. Dos 40 sujeitos da amostra, com idade entre 19 e 24 anos, 28 eram mulheres. Três nunca receberam informação sobre drogas e a maioria teve experiências com consumidores ou consumiram. Esse problema na Costa Rica está associado à estigmatização e ao tráfico. Os sujeitos reconhecem que a responsabilidade é de todos, embora identifiquem contradição do Estado, consentindo com a publicidade do cigarro e sua cumplicidade com as fábricas de bebidas. Reconhecem a importância da prevenção e consideram que a forma de abordar o problema não tem sido adequada. Gostariam que o tema fosse incorporado aos seus estudos, ensinando como atuar e se relacionar com pessoas que consomem drogas.

Descritores: Drogas Desenhadas; Enfermagem; Estudantes de Enfermagem; Ensino.

\section{Experiences and Meanings of the Drugs Phenomenon in Nursing Students}

The aim of this study was to identify the meanings and experiences of nursing students regarding the drugs phenomenon. Data were collected through a semi-structured interview. Data analysis showed five theme categories. Of the 40 subjects in the sample, between 19 and 24 years old, 28 were women. Three had never received information about drugs, and most had had experiences with consumers or had consumed drugs. In Costa Rica, this problem is associated with stigma and traffic. They recognize that everyone is responsible, and consent with the State's contradictory attitude through tobacco company advertisements and complicity with distilleries. They acknowledge the importance of prevention and consider that the current approach of this issue is not appropriate. They would like it to be incorporated in their education, providing strategies on how to act and relate with drugs consumers.

Descriptors: Designer Drugs; Nursing; Students, Nursing; Teaching.

\section{Introducción}

Como los otros países de América Latina, Costa Rica no es una excepción en cuanto al tema de desarrollo. La globalización de la economía mundial y la liberalización de los mercados han tenido gran influencia en la organización socioeconómica costarricense.

La realidad nacional constituye en el presente un crisol de contradicciones que afectan a la población en lo colectivo y en lo individual.

Una de las consecuencias de estas contradicciones sociales es la presencia desproporcionada del fenómeno de las drogas en sus diversas manifestaciones. Un estudio sobre los costos de este problema, en Costa Rica, menciona que en el periodo comprendido entre el 2000 y 2003, estos costos aumentaron en $20 \%$, principalmente debido a la inversión estatal para reducir la oferta, lo cual tiene un gran impacto sobre el ingreso per cápita y el producto interno bruto (PIB). El costo total de las drogas ilegales en Costa Rica para el periodo de estos cuatro años fue de 310 millones de dólares, los cuales se distribuyeron entre costos de atención en salud, pérdida de productividad económica y costos gubernamentales ${ }^{(1)}$. 
En una encuesta nacional del Instituto sobre Alcoholismo y Fármaco-dependencia(2) ${ }^{(2)}$ registró que la prevalencia de consumo activo de alcohol fue de $26 \%$ y que la edad de inicio del consumo se estableció en 17,2 años, y $10,1 \%$ de los sujetos de 12 años y menos ya se han iniciado en el consumo(2).

En el ámbito mundial los datos de la Organización Mundial de la Salud revelan que el consumo de tabaco en países desarrollados y en desarrollo es mayor al encontrado en Costa Rica, pero hay una tendencia al aumento del tabaquismo en las mujeres ${ }^{(3)}$.

Sobre el consumo de tranquilizantes, especialmente benzodiacepinas, la prevalencia del mes es de 0,7\%; la edad de inicio es de 25,4 en el año 2000. Sobre estimulantes, se tiene que la prevalencia del mes es $0,4 \%$, siendo más frecuente entre hombres y el grupo de edad es amplio, entre los 19 y los 40 años(2).

Con respecto a las drogas ilícitas, la marihuana y el crack tienen los mayores niveles de prevalencia, con $0,3 \%$ y un $0,1 \%$, respectivamente. Estas drogas están estrechamente asociadas con la población que está expuesta y experimenta altos niveles de estrés. Los datos encontrados en nuestro país también coinciden a nivel mundial en lo que se refiere a drogas ilícitas; se ha encontrado una alta prevalencia en aquellos países cuyo territorio es apto para el cultivo de esta droga(2).

La incidencia en el consumo de drogas ilícitas en Costa Rica tiende a aumentar, según las estadísticas de los servicios de emergencia de los hospitales nacionales y de la consulta de seguimiento ${ }^{(2,4)}$.

Los datos epidemiológicos sólo muestran una parte muy pequeña del problema. El fenómeno de las drogas ha penetrado en todos los sectores de la vida de las sociedades en el ámbito mundial.

La mayor parte de las investigaciones sobre el tema de las drogas, que centran su interés en estudiantes universitarios, tienen como enfoque el uso o el consumo. A partir de la revisión realizada en las bases de datos LILACS y MEDLINE, se encontró un estudio para validar un instrumento con el propósito de medir el consumo de drogas lícitas e ilícitas en una población universitaria(5). Un estudio sobre uso de drogas y comportamiento de riesgo en una muestra de 200 estudiantes universitarios de primer año de la carrera, al aplicar el cuestionario Youth Risk Behavior Survey (YRBS), concluyó que hay relación entre el uso de sustancias psicoactivas y la recreación y también que el consumo de drogas ilícitas aumentó el comportamiento sexual de riesgo, especialmente en los hombres ${ }^{(6)}$.

Un estudio epidemiológico con 984 sujetos observó que los estudiantes tienen factores protectores como participar en actividades extra académicas, lúdicas y deportivas, vivir con su familia y estrategias personales de afrontamiento, así como un proyecto de vida construido libre y autónomo(7). También sobre consumo se han realizado investigaciones con estudiantes universitarios, en algunos casos relacionados con los conocimientos o actitudes que tienen hacia las drogas ${ }^{(8-10)}$

Investigaciones cualitativas estudiaron las prioridades en cuanto a los valores y el consumo de

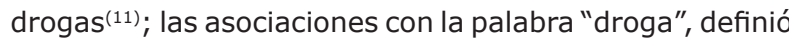
términos valorativos negativos(12); las representaciones sociales de las drogas psicotrópicas ilícitas en estudiantes universitarios destaca que los jóvenes universitarios rechazan el prohibicionismo, la estigmatización y el ocultamiento de la información(13); en Chile se concluyó que es relevante considerar la dimensión simbólica en las reflexiones y acciones relacionadas con las drogas $^{(14)}$; un estudio con el personal de enfermería de una sala hospitalaria encontró que trabajar con drogas psicotrópicas es un trabajo como cualquier otro, que la sustancia psicoactiva tiene posibilidad de ser consumida y que existe el miedo de hablar sobre el tema, considerado asunto prohibido(15).

Un estudio multicéntrico entre estudiantes de enfermería definió ocho categorías de creencias de acuerdo al modelo de Rosenstock: la vulnerabilidad y el entorno; factores de riesgo; daños a la salud; estereotipos; factores protectores; condicionantes; acciones protectoras y saludables; $\mathrm{y}$, comportamientos no saludables ${ }^{(16)}$

En Costa Rica se realizó dos importantes estudios sobre el consumo de drogas, un entre jóvenes con un análisis comparativo de los años 1990, 1995 y 2000, basado en la cuesta nacional del Instituto sobre Alcoholismo y Farmacodependencia( ${ }^{(2,17)}$ y otro sobre la perspectiva crítica de la familia y de personas cercanas sobre factores de riesgo ${ }^{(18)}$.

Conocer y analizar las experiencias y los significados que otorgan al fenómeno de las drogas, las(los) estudiantes de enfermería, es importante para crear e implementar mejores intervenciones.

Así, el objetivo de este estudio fue identificar e interpretar los significados y las experiencias de estudiantes de enfermería sobre el fenómeno de las drogas.

\section{Propuesta teórico metodológica}

Se plantea un estudio interpretativo basado en el método estructural dialéctico, el cual además de 
describir las expresiones de estudiantes, en torno a las experiencias y significados que tienen sobre el fenómeno de las drogas, busca interpretar esa construcción.

\section{Selección de los participantes}

La población que se consideró, fueron estudiantes, hombres y mujeres, del tercer año de la carrera de enfermería de la Universidad de Costa Rica. Esta población era de 120 estudiantes, que en el tercer año cursaban el II módulo Intervención de enfermería con la niñez mórbida, con 20 grupos de seis estudiantes quienes realizaban la práctica en diferentes instituciones hospitalarias del país.

Para la selección, se tomó como criterio de representatividad el equiparar el número de hombres y mujeres en cada grupo, según la población lo permitió. De esta manera, se consideró trabajar con 40 estudiantes. Además se estableció como criterio de exclusión, que estuviesen participando en otra investigación sobre drogas, o que hubiesen realizado algún curso sobre la temática durante los dos últimos años.

\section{Instrumentos}

La técnica para la recolección de la información correspondió a la entrevista semi estructurada, realizada por la investigadora. La elaboración de las interrogantes fueron formuladas a partir de la teoría Ego-ecológica, en la cual la relación de la persona como ser social y su entorno es dialéctica.

La teoría Ego-ecológica permite conocer la identidad psicosocial del individuo, sus peculiaridades y su realidad a través de las representaciones que posee acerca de sí mismo, de sus pares y de los grupos con los cuales se interrelaciona. La realidad exterior se construye $y$ se organiza a partir del ambiente interno y éste refleja la construcción social que la persona ha hecho de su realidad y de su historia, incluyendo lo cotidiano, sus intereses, sus representaciones y significados, reunidos en una memoria funcional que no es percibida(19).

Así, se utilizó un instrumento estructurado con el fin de caracterizar a las personas en términos de datos generales, sociodemográficos y antecedentes sobre el tema de estudio y se platean las frases generadoras de la narrativa como propuestas de discusión que promuevan, en la persona, la reflexión y expresión analógica.

\section{Procedimiento}

Para la selección de los participantes, se solicitó un espacio a la profesora coordinadora del módulo, donde se les explicó a todos los grupos el propósito de la investigación y se les solicitó la participación voluntaria. Posteriormente, se hicieron las citas para la entrevista y se les entregó el consentimiento informado, aprobado en sesión del Comité Científico de la Universidad de Costa Rica No 53, del 03/09/2003.

Las entrevistas se realizaron en un cubículo donde se brinda atención en enfermería en Salud Mental, dentro del edificio de la Escuela de Enfermería.

Los datos se fueron procesados y analizados en forma paralela, para posteriormente proceder a la interpretación de los resultados buscando entrar en la esencia del imaginario del grupo de estudio. Las categorías se fijaron a partir de los datos, buscando los núcleos de significado para la interpretación.

\section{Resultados y discusión}

\section{Características de la muestra}

Entre las características de la muestra de estudiantes de enfermería se destaca que de las 40 personas entrevistadas, 28 eran mujeres y 12 eran hombres. La edad se encontraba entre los 20 y los 24 años y solamente dos personas estaban casados. El lugar de residencia de la mayoría estaba en la gran área metropolitana, mientras que 16 estudiantes vivían fuera de ella y tenían su residencia en San José solamente durante el periodo lectivo. La gran mayoría (37) tenía una beca de la universidad para costear sus estudios y 15 estudiantes refirieron que trabajan en lugares como Mc Donald, con horas como asistentes en la UCR, en un hospital durante los días libres y en las vacaciones, en un centro médico privado y en el parque de diversiones los fines de semana.

Recibían apoyo económico de sus familias 25 estudiantes, en su mayoría mujeres y no recibían este apoyo 13 estudiantes, en su mayoría hombres; dos personas manifestaron que el apoyo no era constante. Nueve personas expresaron que su ingreso no eran suficientes para cubrir sus necesidades.

Sobre el fenómeno de las drogas, 3 manifestaron que nunca habían recibido información sobre este tema. Esta información la recibieron en su mayoría durante los años en que cursaban el colegio o en la universidad en los seminarios de Realidad Nacional en los cursos de humanidades.

Sobre lo que recuerdan de esa información, la mayoría se refirió: al tipo de drogas y sus consecuencias; a factores que provocaban el consumo y riesgos; a la dependencia y las consecuencias fisiológicas y 
psicológicas; a drogas descubiertas recientemente y a medicamentos que crean dependencia; a la diferencia entre drogas lícitas e ilícitas; a la problemática con la juventud en los colegios; y, a la cadena de procesamiento, venta, distribución, productores y consumidores.

\section{Significados y experiencias previas}

Los significados que las personas asignan a los diferentes fenómenos sociales pueden ser interpretados sólo si se considera el contexto general y particular en que dicho fenómeno sucede. La construcción que las(los) estudiantes de enfermería hacen sobre el fenómeno de las drogas está influenciado principalmente por su experiencia de vida, más que por el estudio del problema en su formación profesional(6,10,15).

El fenómeno de las drogas, para las(los) estudiantes de enfermería, tiene un significado negativo, con una visión catastrófica, representada en forma de deterioro social y lo relacionan con otros problemas sociales como prostitución, desempleo y personas que viven en zonas marginales.

La visión del problema presenta algunas contradicciones, al expresar que es una situación muy común, pero que está escondida, que está carcomiendo a la sociedad, que es muy difícil salir del problema porque finalmente destruye como un cáncer mundial. Consideran que el consumo de las drogas, en parte, viene a llenar los vacíos en las persona, pero que es un círculo que las encierra del cual es muy difícil salir.

Todas las personas consultadas, excepto una, habían conocido a alguna persona que había consumido droga - la estuviese consumiendo. Las experiencias cercanas son bastante frecuentes, en relación a familiares y a amigos o compañeros del colegio y de la universidad. Los relatos coinciden en que las personas que consumen por lo general han tenido muchos problemas, los cuales generalmente tienen que ver con la relación familiar.

La experiencia de entrar en contacto con el fenómeno se inicia en el colegio. Este es el principal referente y consideran que lo que sucede en estos centros con la droga es muy grave. Sin embargo cuando la experiencia acontece en la universidad no la ven tan grave, hacen referencia a los bares y fiestas, a la presión por el estudio y a que las personas ya saben lo que hacen. Cuando la referencia se da en términos del ambiente universitario la visión del fenómeno cambia y tiende a verse menos catastrófica. El grupo coincide en que la madurez que se espera en las(los) estudiantes de la universidad debe marcar la diferencia, esto porque en la secundaria la presión de grupo es muy fuerte. También hacen referencia a que la atracción por lo desconocido y el deseo de experimentar se presenta con frecuencia en los grupos de colegiales: entonces lo desconocido o prohibido se hace más interesante que lo que no lo es.

En el colegio, se da un fenómeno de discriminación en lo cotidiano de la relación entre pares y de éstos con las personas adultas, se llega a marginar a quien consume drogas, hasta el punto de que estas personas se empiezan a unir en una especie de contracultura, sin embargo señalan que: el grupo que consumía drogas ilícitas lo hacía aparte, se separaba del resto y no generaban problema.

Cuando se refieren a personas adultas especialmente a miembros de su familia, mencionan que el consumo es de alcohol y tabaco. Cuando hacen referencia al ambiente universitario, señalan que si es frecuente el consumo de marihuana. Sin embargo, del grupo que participó en esta investigación muy pocos hicieron referencia explícita al problema como consumidores, y cuando lo hicieron se refirieron al consumo de alcohol y tabaco, en ningún caso expresaron el uso de drogas ilícitas, aunque si quedó manifiesto que este grupo de estudiantes frecuentan las fiestas y bares, como lo hacen otros grupos de estudiantes de la universidad.

En varios casos asumen la responsabilidad de otra persona que consume drogas, expresan que hicieron algo para que la persona dejara el consumo, expresaron experiencias dolorosas, aunque manifestaron una tendencia a reafirmar que eso no los afectó.

En algunos relatos, hay una mayor relación afectiva con la persona que consume, especialmente drogas ilícitas. Sin embargo, es una constante que durante la entrevista, la(el) estudiante establezca un distanciamiento con el fenómeno. A pesar de esto, se encuentra en algunos casos cierta decepción porque la(el) estudiante de enfermería sintió la obligación de que debía hacer algo por la persona. Esto generó sentimientos de culpa y frustración, asociados a la pérdida de amigos.

\section{Sobre las causas}

Las personas que consumen drogas les generan lástima, porque lo hacen para desahogarse o ser aceptados, refieren que les cuesta entender los motivos pero en general consideran que no los discriminan. En varios relatos se manifestó el miedo, ya que el problema es muy grande y si la persona vende la drogas, esto les puede perjudicar o tener problemas con la policía.

La familia está presente con frecuencia en las respuestas e insisten en que esta es fundamental para 
prevenir el consumo y que en algunos casos, la adicción es el reflejo de lo que sucede en la casa. Por otra parte, hacen referencia al impacto que tiene este fenómeno porque en los casos que mencionan, se expresa el sufrimiento de los padres, abuelos, hijos e hijas. También señalan la negación que hace la familia del problema y la dificultad que tienen para resolverlo.

Otra situación que motiva a las personas a consumir drogas, según la opinión de este grupo de estudiantes, es la presión social y el excesivo estrés; por otra parte, en la realidad en que viven no cuentan con personas que les brinden ayuda.

Aunque no es frecuente, en algunas respuestas se refirieron a la difícil situación socioeconómica y a la pobreza como la causa del consumo de drogas. Reconocen que existen estereotipos hacia estas personas que han logrado sacar una carrera a pesar de que frecuentemente usan drogas.

\section{El fenómeno de las drogas en estudiantes de enfermería}

En varias respuestas, el problema del consumo es serio, aunque solamente se refieren al consumo de drogas lícitas, muy esporádicamente mencionan drogas ilícitas, aunque no se especifican cuales.

La carrera genera mucha presión, a pesar de contar con una beca tienen que recurrir en muchos casos a trabajar durante el tiempo que les queda libre, el cual debería invertirse en preparar sus trabajos y estudiar ${ }^{(6,10,15)}$.

Para muchos estudiantes, entrar a la universidad es un cambio radical, los patrones culturales son otros y rápidamente se incursiona en un mundo donde la persona se siente más libre y responsable de sí misma. El uso de drogas en el ambiente universitario está asociado con las formas de vida y con las formas de recreación de las(los) jóvenes (6-9).

Hay también una cierta indiferencia ante el problema o éste se evalúa como siendo "pequeñito", sin ver su magnitud, que ha aumentado y es tan complejo que la población tiende a no verlo. Esto representa una reclamación en el sentido de que sucede lo mismo en el grupo de estudiantes de enfermería.

Se reconoce que estudiar enfermería ofrece la posibilidad de conocer los efectos que el consumo de drogas tiene sobre la salud. La imagen que la persona que estudia enfermería presenta a la sociedad se pude ver perjudicada si esta persona consume drogas.

Una idea generalizada es que, saber sobre este tema es fundamental, porque la práctica en enfermería exige atender diferentes grupos poblacionales $y$ en algunos hay mayor incidencia de adicciones.

\section{Sobre la responsabilidad ante el fenómeno}

Además de considerar el consumo de drogas como un problema amplio y complejo, los estudiantes señalan que la responsabilidad es de todos y mencionan la responsabilidad que tiene el estado. Existe tolerancia en cuanto a la publicidad de las compañías nacionales y extranjeras que producen estas sustancias. El estado es empresario en la producción de alcohol, y el mismo gobierno hace la observación de que se debe consumir con moderación.

Por otra parte, en Costa Rica, los accidentes de tránsito, en los que existe consumo de alcohol, se ubican entre las primeras causas de muerte y discapacidad. Los relatos indican que las autoridades responsables de atender este fenómeno en nuestro país, saben en donde está el problema pero no lo abordan directamente(2).

En relación al desarrollo individual se señala con frecuencia el papel de la socialización primaria, según las respuestas, la familia falla y las presiones sociales entran en juego conforme el niño y la niña crece. Así mismo, consideran que la adolescencia es la etapa de mayor vulnerabilidad(17).

Aunque no establecen con claridad la relación entre los determinantes socioculturales, políticos y económicos con el problema del consumo, los estudiantes mencionan que el estado tiene la responsabilidad de disminuir la vulnerabilidad y la demanda.

\section{Cómo se está abordando el problema}

En Costa Rica el fenómeno de las drogas está asociado a la estigmatización y discriminación de las personas adictas, mientras que el tráfico es cada vez más fuerte, especializado y lucrativo(1-2,17-18).

Según las respuestas expresadas por las(los) estudiantes, las estrategias que se han implementado para abordar el problema son muy débiles y no existe relación entre la magnitud del problema y los recursos que se invierten para afrontarlo.

En cuanto a las estrategias para la prevención se hace referencia especialmente a la información, capacitación y actividades educativas, dirigidas a la población joven.

\section{El fenómeno de las drogas en el plan de estudios}

En todas las entrevistas se identificó la idea de que el tema de las drogas es muy débil en el plan de 
estudios, por esto, señalan que debe impartirse durante toda la carrera e iniciarse en los primeros años.

Como un aspecto necesario en su formación, expresan que es necesario aprender a interpretar los mensajes publicitarios y estudiar las condiciones del entorno asociadas a la presión social e ideológica, especialmente la que va dirigida a la población joven. Esto va asociado a la idea de desarrollar habilidades para trabajar en participación social(10).

El trabajo en la comunidad lo plantean como prioritario y manifiestan la necesidad de contar con metodologías participativas para promover la prevención.

Les preocupa mucho la forma en que van a relacionarse, lo cual nos lleva a pensar que además de la prevención, consideran fundamental trabajar en aspectos de atención y rehabilitación y entonces, señalan la importancia de enfocarse en la persona y no sólo en el problema.

El resultado de esta investigación, finalmente reveló un elemento fundamental, el cual correspondió a la aceptación que tiene el tema en este grupo de estudiantes. Para la mayoría es un tema que va a complementar su carrera. Sobresale el hecho de que muestran tener claridad en lo que quieren y lo que necesitan para integrar el tema del fenómeno de las drogas en el plan de estudios de la carrera.

\section{Consideraciones conclusivas}

La condición económica de las(los) estudiantes, quienes con frecuencia tienen que trabajar, así como la presión de la carrera, y el ambiente social paralelo a la universidad se convierten en factores de riesgo para el consumo de drogas, por los niveles de estrés que requieren manejar, además de la presión social.

Aunque la mayoría ha recibido algún tipo de información, sea en el colegio o en la universidad, se interpreta que no están satisfechos con ese conocimiento.

El significado del fenómeno de las drogas tiene una connotación catastrófica con mucha ambivalencia y discriminación. Cuando la referencia se da en términos del ambiente universitario la visión del fenómeno cambia y tiende a verse menos catastrófica.

Los estudiantes de enfermería procuran un distanciamiento con el consumo. A pesar de esto, se encuentra en algunos casos cierta decepción porque la(el) estudiante de enfermería sintió la obligación de que debían hacer algo por la persona. Esto generó sentimientos de culpa y frustración por la pérdida de amigos.
Consideran que la persona adicta es la responsable y que no se puede hacer nada, si ella no quiere salir del problema, porque el problema es complejo.

En cuanto al acercamiento cognitivo hay una tendencia a explicar el problema desde las carencias que tiene la persona: familiares, conflictos, marginalidad, exclusión, presión social y excesivo estrés.

En el aspecto relacional y afectivo no se separa el fenómeno de las drogas de las experiencias personales, ya que la mayoría había tenido algún acercamiento con personas adictas.

Para referirse a la propia participación en esta experiencia, se dieron dos polos de respuesta: en primer lugar como persona, y posteriormente como estudiante de enfermería. La imagen de la profesión se puede ver perjudicada si la persona que estudia enfermería consume drogas. La mayoría de los entrevistados coincide en que la enfermería es una disciplina que debe atender a esta problemática. Señalan que en la escuela de enfermería faltan cursos sobre el tema y que en general es un tema del que se habla poco.

Facilidades y dificultades: el hecho de formar parte del grupo docente de la escuela facilitó la ejecución de la investigación pero no aseguró la disposición de las(los) estudiantes a participar.

\section{Agradecimientos}

Agradecemos a la Comisión Interamericana para el Control del Abuso de Drogas/CICAD de la Secretaria de Seguridad Multidimensional/SSM de la Organización de Ios Estados Americanos/OEA, la Secretaria Nacional de Políticas sobre Drogas/SENAD do Gabinete de Seguridad Institucional/Brasil, la Escuela de Enfermería de Ribeirao Preto de la Universidad de Sao Paulo y Centro Colaborador de la Organización Mundial de la Salud para el Desarrollo de la Investigación en Enfermería, la población representada en los estudios de investigación, bien como a las autoridades de las universidades representadas por los participantes del Programa En-Line de Especialización en Investigación sobre el Fenómeno de las Drogas, periodos 2006, 2007, 2008 y 2009.

\section{Referencias}

1. Gómez AP, Zapata JEV, Sickert CR. Informe consolidado sobre los costos del problema de las drogas ilegales en Costa Rica: 2000-2003. Costa Rica: CICAD. [acesso 05 maio 2010]. Disponível em: http://www.cicad.oas. org/oid/new/research/coasts/informe\%20Final\%20 Costa\%Rica.pdfs. 
2. Instituto de Alcoholismo y Fármacodependencia (IAFA), Costa Rica. Una aproximación a la realidad nacional. San José. Costa Rica: IAFA; 2004.

3. Luis MAV, Pillón SC. Epidemiología del uso de las drogas. En: Módulo II. El fenómeno de las drogas en América y en el Mundo. Curso de Capacitación sobre Investigación del fenómeno de las drogas. Ribeirão Preto (SP): SENAD: CICAD/OEA; 2006.

4. Instituto de Alcoholismo y Fármacodependencia (IAFA), Costa Rica. Encuesta sobre juventud y drogas. San José. Costa Rica: IAFA; 2006.

5. Rodríguez TJ, Hernández AE. Terapia psicológica. Psicol Clín Chile. 2005;23(1):83-90.

6. Pillon SC, O'brien B, Chavez KAP. La relación entre uso de drogas y comportamiento de riesgo en estudiantes universitarios de Brasil. Rev. Latino-Am. Enfermagem. 2005;13(n. esp):1169-76.

7. Téllez J, Cote M, Savogal F, Martínez E, Cruz U. Factores de riesgo y consumo de drogas en estudiantes universitarios. Rev Fac Med Bogotá. 2003;51(1):15-24.

8. Castro CAJ, Pinilla MI. Estudio sobre consumo y conocimiento de las drogas en una población de estudiantes universitarios en la ciudad de Bogotá. Rev Esc Colombiana Med. 2002;7(2):68-75.

9. Carraro TE, Rassool GH, Luis MAV. La formación del enfermero y el fenómeno de las drogas en el sur de Brasil: actitudes y creencias de los estudiantes de enfermería sobre la atención. Rev. Latino-Am. Enfermagem. 2005;13 (n.esp):863-71.

10. Mendonza EV, Pillon SC. La formación de enfermeras y el fenómeno de las drogas: conocimientos, actitudes y creencias. Rev. Latino-Am. Enfermagem. 2005;13(n. esp):845-53.

11. Tamayo A, Nicaretta M, Ribeiro R, Barbosa LPG. Prioridades axiológicas y consumo de drogas. Acta Psiq Psicol Am Latina. 1995;41(4):300-7.

12. Stein RL. Asociaciones libres al término "droga" en dos grupos de estudiantes universitarios Psicoactiva. Centro de Información y Educación para la Prevención del Abuso de Drogas - CEDRO 1987;(1):55-64.

13. Bustos SS. Representaciones sociales de sustancias psicoactivas ilícitas en universitarios de la ciudad de Santiago. Cuad Méd Soc. 2005;45(2):117-37.

14. Martins ERC, Corrêa AK. El trabajo con sustancias psicoactivas: significado que tiene para el trabajador de enfermería. Rev. Latino-Am. Enfermagem. 2004;12(n. esp):398-405.

15. Rebolledo NO, Costa MCS. Significados y contradicciones del fenómeno de las drogas: drogas lícitas e ilícitas en Chile. Rev. Latino-Am. Enfermagem. 2005;13(n. esp.):903-11.
16. Medina NMO, Rebolledo EAO, Pedrão LJ. El significado de drogas para el estudiante de enfermería según el modelo de creencias en salud de Rosenstock. Rev. Latino-Am. Enfermagem. 2004;12(n. esp):316-23.

17. Bejarano J. Encuesta sobre percepciones y consumo de drogas en colegiales. Costa Rica: IAFA - Instituto sobre Alcoolismo y Farmacodependencia; 2006.

18. Hernández MG, Brands B, Adlaf E, Giesbrecht N, Simich L, Wright MGM. Pespectiva critica de la familia y de personas cercanas sobre factores de riesgo familiares y comunitarios en el uso de drogas ilícitas en San José, Costa Rica. Rev. Latino-Am. Enfermagem. 2009;17(n. esp):770-5.

19. Mello R, Furegato ARF. A Teoria Ego-Ecológica como método de compreensão do sujeito em seu contexto histórico-social. In: $13^{\circ}$ Seminário Nacional de Pesquisa em Enfermagem, 2005; São Luis (MA); 2005. p. 1-3. 